\title{
Uso de fuentes históricas en formación inicial de profesores
}

\begin{abstract}
Resumen
La didáctica de la Historia debe responder a la formación de los futuros profesores de esta especialidad con el fin de reconocer las dificultades que enfrenta la escuela en este ámbito. El objetivo principal de esta investigación es construir conocimientos en torno al uso de fuentes históricas en la carrera de Pedagogía en Historia. Se enmarcó en un paradigma interpretativo, con una orientación hermenéutica y un enfoque metodológico descriptivo-cualitativo. Se concluyó que el uso de fuentes históricas influye positivamente en el fortalecimiento del pensamiento histórico y que su uso constituye un recurso didáctico valioso para enseñar el pasado.
\end{abstract}

\section{Palabras claves}

Práctica pedagógica; enseñanza de la historia; método histórico; formación de profesores

\section{Transferencia a la práctica}

Los resultados de esta investigación contribuyen a discutir sobre la importancia del uso de fuentes históricas en la formación inicial de profesores de historia en Chile. Se las posiciona como el recurso didáctico imprescindible para enseñar la disciplina histórica en el aula, lo cual favorece tanto la formación del pensamiento histórico en estudiantes universitarios de pedagogía como su posterior desempeño en el proceso de enseñanza de esta materia para acercar así a niños y jóvenes a la comprensión del pasado.

Para citar este artículo / To cite this article / Pour citer cet article / Para citar este artigo

Montanares-Vargas, E. \& Llancavil-Llancavil, D. R. (2016). Uso de fuentes históricas en formación inicial de profesores. magis, Revista Internacional de Investigación en Educación, 8 (17), 85-98. http://dx.doi.org/10.11144/Javeriana.m8-17.ufhf 
Keywords

Teaching practice; history teaching;

historical method; teacher development

\section{Mots clés}

Pratique pédagogique; enseignement de I'histoire; méthode historique; formation de professeurs

\section{Abstract}

Teaching history must respond to the teaching development of future teachers ficulties faced by schools in this area. This research aims to build knowledge abou the use of historical sources in a bachelor's degree in history education. This research is framed within the interpretive paradigm, with a hermeneutic orientation and a descriptive-qualitative methodological approach. We conclude that the use of historical sources has a positive effect in the strengthening of historical thinking and that its use is a valuable educational resource for teaching the past. of this field in order to recognize the dif-

\section{Transfer to practice}

The results of this research contribute to the debate about the importance of using historical sources in the initial teacher development of history teachers in Chile. Historical sources are considered as an essentia educational resource for teaching history in the classroom. Using historical sources promote the development of historical thinking in undergraduate students and facilitate their subsequent performance as history teachers in order to encourage children and youth to understand past events.

\section{Transfert à la pratique}

\section{Résumé}

La didactique de I'Histoire doit répondre à la formation des futurs professeurs de cette spécialité avec l'objectif de reconnaitre les difficultés qui souffre l'école dans ce domaine. L'objectif principal de cette recherche est celui de construire les connaissances par rapport à l'usage de sources historiques dans les études de Pédagogie en Histoire. La recherche se situe dans le paradigme interprétatif, avec une orientation herméneutique et une perspective méthodologique descriptive-qualitative. On conclut que l'usage de sources historiques contribue positivement dans le renforcement de la pensée historique et les utiliser constitue un recours didactique précieux pour l'enseignement du passé.

Les résultats de cette recherche contribuent au débat sur l'importance de l'usage de sources historiques dans la formation initiale de professeurs d'histoire au Chili. On les situe en tant que le recours didactique incontournable pour l'enseignement de la discipline historique dans la salle de classe, cela aide tantôt dans la formation de la pensée historique chez les étudiants universitaires de pédagogie que dans leur postérieure exercice dans le processus d'enseignement de cette matière et ainsi rapprocher aux enfants et aux jeunes à la compréhension du passé.

\section{Palavras chave}

Prática pedagógica; ensino da história; método histórico; formação de professores

\section{Resumo}

A didática da história deve responder à formação dos futuros professores desta especialidade com o fim de reconhecer as dificuldades que enfrenta a escola neste âmbito. O objetivo principal desta pesquisa é construir conhecimentos em torno do uso de fontes históricas no curso de graduação de pedagogia em história. Estabeleceu-se um paradigma interpretativo, com uma orientação hermenêutica e um enfoque metodológico descritivo-qualitativo. Concluiu-se que o uso de fontes históricas influi positivamente no fortalecimento do pensamento histórico e que seu uso se constitu num recurso didático valioso para ensinar o passado.

\section{Transferência à prática}

Os resultados desta investigação contribuem a discutir sobre a importância do uso de fontes históricas na formação inicial de professores de história em Chile. Posiciona-as como o recurso didático imprescindível para ensinar a disciplina histórica no sala, o qual favorece tanto a formação do pensamento histórico em estudantes universitários de pedagogia como seu posterior desempenho no processo de ensino desta matéria para acercar assim a meninos e jovens ao entendimento do passado. 


\section{Introducción}

Uno de los objetos centrales de estudio de la didáctica de la historia es dar respuesta a la formación de los futuros profesores de esta especialidad, discutiendo sobre los aspectos más relevantes y las principales debilidades que hay en el aula en relación con la enseñanza de esta disciplina. La existencia de un discurso unilateral con limitadas oportunidades para la participación activa, afecta la comprensión y el valor que la Historia tiene para los estudiantes (Pagès i Blanch, 2003). Por esta razón, en la formación inicial se deben realizar cambios significativos en la manera de formar a los futuros profesores.

Muchos estudios convergen en la importancia del uso de fuentes escritas, primarias o secundarias, como principal herramienta de enseñanza en el aula. Las fuentes históricas constituyen la materia prima del historiador, "múltiples documentos escritos y no escritos a partir de los cuales puede conocer y construir una explicación sobre el pasado y los modos que sus huellas acontecen (o ya no) en el presente" (Meschiany, 2013, p. 44).

Peter Lee y Rosalyn Ashby (2000) se refieren a la enseñanza con fuentes como una necesidad, ya que la historia es una disciplina que tiene sus propios procedimientos y estándares para elaborar conocimientos válidos sobre el pasado y sus múltiples relaciones con el presente. Estas vinculan al estudiante con el pasado, y como lo explica Sam Wineburg (2001), las diferencias en las formas de vida entre el pasado y el presente cobran significado mediante el uso de fuentes originales, y permite a los estudiantes imaginar un mundo con un marco moral distinto al de hoy. Esto último es uno de los aspectos más complejos con los que se enfrenta un profesor, y forma parte del llamado pensamiento histórico, entendido como el desarrollo del pensamiento crítico en relación con documentos del pasado, opiniones de los actores de la historia y discursos, entre otros (Éthier, Demers \& Lefrançois, 2010). Autores como Augusta Valle-Taiman (2011) agregan que enseñar con fuentes no solo aproxima al estudiante a la naturaleza del pensamiento histórico sino a la labor del historiador. De esta manera, el contacto inicial con las fuentes históricas permite comprender la forma en que el conocimiento sobre el pasado llega al presente, independiente de cuán lejano parezca. Sobre lo mismo, Antoni Santisteban-Fernández reconoce que por medio de estas se incorpora la experiencia histórica a la enseñanza (Santisteban, 2010).

De este modo, es fundamental integrar el uso de fuentes históricas en los procesos de formación inicial con el objeto de que los futuros profesores desarrollen ellos mismos estas habilidades y valoren la importancia del uso habitual de estas en el trabajo que realizarán con sus estudiantes.

La presentación de este artículo se ha organizado en cuatro apartados. En el primero, se analizan los referentes conceptuales; en el segundo, se describe la metodología teniendo en cuenta la sistematización del proceso alcanzado; en el tercero, se exponen los resultados, y en el cuarto, se hace la discusión y se sacan las conclusiones.

\section{Referentes conceptuales}

\section{Didáctica de la historia}

En la actualidad, la didáctica de la historia tiene un espacio protagónico en los programas de formación de profesores de esta disciplina. Constituye uno los saberes asociados a la competencia profesional del futuro profesor y hay cursos destinados a su enseñanza que se suman a las cátedras pedagógicas y disciplinarias. "La didáctica de la historia se cons-

\footnotetext{
Descripción del artículo | Article description | Description de I'article | Artigo descrição

Este artículo de investigación aborda la temática de la enseñanza de la Historia que usa fuentes históricas, en el marco de la formación inicial de profesores. Se discute sobre la importancia de enseñar la disciplina histórica en profundidad en el aula, cuestión que debería mejorar significativamente los procesos de aprendizaje en la disciplina.
} 
tituye en un espacio de síntesis y de integración de la formación disciplinar específica y de la formación pedagógica, de construcción de herramientas teóricas y metodológicas para el desempeño en la práctica docente..." (Andelique, 2011, p. 262).

En el proceso de formación del futuro profesor de historia están involucrados diversos conocimientos que este requerirá para llevar a cabo la enseñanza de la disciplina cuando deba enseñar. Así: "Para ser profesor o profesora no basta con saber lo que debe enseñarse, es necesario saber muchas otras cosas. Sin duda, nadie puede enseñar lo que no sabe. En consecuencia, para enseñar es necesario saber pero no basta con solo saber para saber enseñar" (Pagès i Blanch, 2003, p. 157). Lee S. Shulman (1987-2005) sostiene que el conocimiento base de un profesor debe incluir al menos siete categorías de conocimientos diferentes: conocimiento del contenido, conocimiento didáctico general, conocimiento curricular, conocimiento didáctico del contenido, conocimiento de las características de los estudiantes, conocimiento de los contextos educativos y conocimiento de las finalidades educativas. Esto supone que no basta con que el profesor domine la materia a enseñar sino que también requiere otros conocimientos, y en particular cómo enseñar un contenido histórico. En este sentido, la didáctica de la historia se ocupa de enseñar a enseñar historia, de formar como profesores de la disciplina a los estudiantes de pedagogía en historia.

Los procesos de prácticas a los que se ven enfrentados los futuros profesores de historia, evidencian problemáticas como la falta de interés de los alumnos por la asignatura, la memorización y la reproducción de información, el tratamiento positivista y político de los contenidos, la carencia de una variedad de estrategias de enseñanza, las limitaciones en el desarrollo de un pensamiento reflexivo y crítico en los estudiantes, las dificultades en la comprensión de conceptos propios de la disciplina como el tiempo histórico y la ausencia de métodos similares a los empleados por el historiador (Andelique, 2011; Prats, 2000; Suárez, 2010). Frente a esa realidad, la didáctica de la historia debe proponer alternativas de enseñanza, generar recursos y estrategias metodológicas innovadoras que propicien el aprendizaje de la disciplina y posibiliten que los estudiantes asuman un papel activo, crítico y creativo con respecto a su realidad, junto con prepararlos para que se sitúen en el mundo, sepan interpretarlo desde su historicidad e intervengan en él con conocimiento de causa y sean protagonistas del devenir histórico (Pagès i Blanch, 2003; Perafán, 2013; Prieto, Gómez \& Miralles, 2013).

Los futuros profesores de historia resultan actores relevantes para reorientar la actual enseñanza de la disciplina en educación primaria y secundaria. "Los maestros de historia deben ir más allá de hacer historia o pensar históricamente para sí: deben poder ayudar a otros a aprender historia y a aprender a pensar históricamente" (Bain, 2005, p. 5). Frente a esto, la didáctica de la historia debe entregar las herramientas necesarias para que los profesores de historia enseñen a pensar históricamente a sus estudiantes. Esto se logra fundamentalmente a partir del trabajo permanente con fuentes históricas primarias y secundarias.

\section{Fuentes bistóricas}

La enseñanza y el aprendizaje del pasado se ven limitados por la dificultad para contextualizar los hechos ocurridos en este. A diferencia de otras disciplinas, la historia se interesa más por el significado de los hechos que por los hechos en sí mismos. Frente a esto, se requiere una comprensión de cómo se llega al conocimiento del pasado para así poder emitir una explicación sobre el porqué ocurrieron los hechos de una determinada 
forma. Todo lo anterior lleva a la selección de determinadas estrategias, por parte del docente, para desarrollar aquella comprensión en los estudiantes. En ese mismo sentido, resulta relevante el trabajo con fuentes históricas y enseñar a los alumnos a pensar como historiadores (Wineburg, 2001). Las fuentes históricas primarias constituyen la materia prima por excelencia para el conocimiento y comprensión de la historia. "Se trata de aquellas que fueron producidas al paso mismo de los acontecimientos de los cuales nos informan y son variadísimas" (Prats \& Santacana, 2011a, p. 77). La tabla 1 presenta los tipos de fuentes primarias.

Tabla 1

Tipos de fuentes primarias

\begin{tabular}{l|l}
\hline Tipo de fuente & Descripción \\
\hline Fuentes materiales & $\begin{array}{l}\text { Edificios, caminos, instrumentos, vestidos, armas, } \\
\text { monumentos y todo tipo de objetos variados de uso } \\
\text { cotidiano (instrumentos de trabajo, de higiene, muebles, } \\
\text { vestidos, etc.) son los que mayor significación cobran en } \\
\text { el trabajo didáctico. }\end{array}$ \\
\hline Fuentes escritas & $\begin{array}{l}\text { Cartas, tratados, crónicas, documentos legales, etc., } \\
\text { son una de las bases más importantes sobre las que } \\
\text { se construye la historia. Entre ellas están también las } \\
\text { periodísticas: prensa, revistas y material gráfico. }\end{array}$ \\
\hline iconográficas & $\begin{array}{l}\text { Grabados, cuadros, dibujos, etc., son abundantes y el } \\
\text { profesorado las tiene siempre a su alcance. Sin embargo, } \\
\text { la mayoría de las veces las utilizamos como meras } \\
\text { ilustraciones, sin entrar en el análisis de sus contenidos. }\end{array}$ \\
\hline Fuentes orales & $\begin{array}{l}\text { A menudo poco utilizadas y son, sin embargo, } \\
\text { importantes para la historia reciente: registrar la voz del } \\
\text { abuelo que nos explica cómo trabajaba, cómo se divertía, } \\
\text { qué hizo en determinada efeméride, cómo transcurrían los } \\
\text { días de fiesta durante su juventud, etc. }\end{array}$ \\
\hline \hline
\end{tabular}

Fuente: elaboración propia, a partir de Joaquín Prats \& Joan Santacana (2011a)

El trabajo directo con fuentes históricas propicia en los estudiantes, entre otros aspectos, una aproximación a la naturaleza del conocimiento histórico y a la labor del historiador. La evidencia empírica demuestra que su uso en el aula favorece, en los niños y jóvenes, la incorporación de la experiencia histórica, el desarrollo de competencias como la interpretación histórica y el pensamiento crítico, así como la capacidad de plantear problemas, elaborar hipótesis, argumentar y aprender procedimientos propios de la disciplina y su correspondiente conceptualización (Fuentes, 2004; Prats \& Santacana, 2011a, 2011b; Rostan, 2002; Valle, 2011; Elizabeth Anne Yeager, Stuart J. Foster, Sean D. Maley, Thom Anderson \& James W. Morris, 1998, en Barton, 2010). En este sentido, los alumnos acostumbrados a trabajar con testimonios o fuentes históricas en el aula tienen percepciones más completas, no solo del historiador, sino también de la disciplina histórica. Por todo lo anterior "el uso de fuentes en las aulas tiene un elevado potencial cognitivo por lo que debería plantearse a lo largo de la educación básica, de primaria a secundaria" (Sáiz, 2014, p. 84). Sin embargo, su tratamiento debe adecuarse a la edad y nivel de desarrollo de los estudiantes ya que los niños pequeños disponen inicialmente de un marco muy poco informado dentro del cual relacionarlos y su experiencia para el análisis es mucho más limitada. Sin embargo, pueden 
comprender conceptos y desarrollar técnicas propias del historiador. Por todo lo anterior, se les debe ayudar a ser conscientes de lo que significa ser un historiador, a analizar el modo en que un historiador examina el material (Pluckrose, 2002).

\section{Pensamiento histórico}

La formación del pensamiento histórico es uno de los objetivos fundamentales en la enseñanza de la historia. El pensamiento histórico confiere a los alumnos habilidades que les permiten abordar el estudio de la historia y así construir su propia representación del pasado y juzgar los hechos históricos. Pensar históricamente requiere tener conciencia de la temporalidad, capacidad para la representación histórica, imaginación histórica e interpretación de las fuentes históricas (Santisteban, 2010). De esa forma, la introducción del método y las técnicas del historiador en la enseñanza de la historia son elementos claves para desarrollarlo. Desde la educación primaria, los estudiantes van progresando en su capacidad de pensar históricamente al adoptar una perspectiva temporal y lograr una empatía histórica, una comprensión del método histórico mediante la interpretación de las fuentes (Éthier, Demers \& Lefrançois, 2010).

Las investigaciones sobre el aprendizaje del pensamiento histórico más recientes han sido recopiladas, entre otros, por Keith C. Barton (2010), Joan Pagès i Blanch (2009) y Rodrigo Henríquez-Vásquez (2011). A partir de estos estudios, se llega a establecer que "el desarrollo del pensamiento histórico es el conjunto de procesos que permiten significar el pasado a través del uso deliberado de estrategias creadas por los historiadores como son el tiempo histórico, la causalidad, la objetividad y la intertextualidad" (Sebastián Plá, 2005, en Henríquez, 2011, p. 2).

Ayudar al desarrollo del pensamiento histórico en los alumnos significa que la enseñanza debe profundizar más en problemáticas y cuestionamientos que lleven a la reflexión -es decir, a un modo de razonar-y menos en contenidos cronológicos, en donde los acontecimientos se suceden uno tras otro sin contextualización ni análisis sino solo memoria. Pensar históricamente es construir de manera crítica, reflexiva, el conocimiento de un fenómeno de estudio en su temporalidad y espacialidad, como indica Antoni Santisteban-Fernández (2010).

En la investigación que se presenta en este artículo se abordan las preguntas: ¿cómo construir conocimientos en torno a la enseñanza de la historia usando fuentes históricas?, ¿cuál es la influencia del uso de fuentes históricas en la formación inicial de estudiantes de pedagogía en historia? Y finalmente: ¿incide la enseñanza de la historia en el desarrollo del pensamiento histórico?

\section{Alcances y límites de la investigación}

En esta investigación se presentan resultados de la actividad didáctica en el aula con estudiantes universitarios que tuvo por objetivo la construcción de conocimiento a partir del uso de fuentes históricas. Estas son fundamentales para el desarrollo del pensamiento histórico y las habilidades asociadas a este en estudiantes de todos los niveles (Wineburg, 2001). En este artículo se comparten los resultados de una experiencia didáctica en un contexto universitario, lo cual permite reconocer esta manera de enseñar historia como una prioridad en la formación inicial de profesores.

Es importante ser cautos en la generalización de sus resultados, debido al tamaño y la especificidad de la muestra. Adicionalmente, se requiere profundizar en esta investigación y aplicar otras técnicas de recogida de datos como entrevistas semiestructuradas con el fin de ahondar en el logro de los objetivos.

\section{Metodología}

Para este estudio se consideró el paradigma interpretativo, orientado hacia una investigación hermenéutica, comprendida la hermenéutica como una actividad de reflexión en el sentido etimológico del término, e igualmente como una actividad interpretativa que permite la captación plena del sentido de los textos en los diferentes contextos por los que ha atravesado la humanidad (Arráez, Calles \& Moreno, 2006).

Al paradigma interpretativo de investigación seleccionado corresponde el abordaje metodológico descriptivo-cualitativo, referido a estudios de los cuales emergen datos descriptivos, que derivan de las palabras de las personas — habladas o escritas - y de su conducta observable. En definitiva, "es un modo de encarar el mundo empírico" (Steven Taylor \& Robert Bogdan, 1996, en Ruedas, 2012).

De esta forma, se pone énfasis en el desarrollo subjetivo e individual de los sujetos, lo que permite la observación de su desempeño durante todo el transcurso de la actividad, con un momento de intervención cuando se espera que hayan alcanzado los aprendizajes.

Participaron 35 estudiantes del curso Didáctica de la Historia, ubicado en el cuarto semestre de la carrera de Pedagogía en Historia, Geografía y Ciencias Sociales, de la Universidad Católica de Temuco. Es un curso mixto con un promedio de 20 años de edad. La universidad es confesional católica y tiene sede en la ciudad de Temuco, Chile.

Al tratarse de una investigación cualitativa cuya sustancia es indagar en la compleja condición humana, adscribe a los principios de la Declaración de Helsinki (AMM, 1964) y destaca particularmente aspectos 
relativos al consentimiento informado, la selección equitativa de los sujetos y la validez (Botto, 2011).

Los instrumentos utilizados fueron los siguientes: (1) trabajo de retroalimentación que dio la posibilidad de seguir el avance de los estudiantes; (2) estados de avance de productos que realizaron los estudiantes durante el proceso en el diario de campo del profesor; (3) talleres de lectura y entrega de matrices con citas de autores referidas a la temática; (4) presentación final de la actividad realizada por los estudiantes; y (5) rúbrica durante la revisión final de la actividad. En la tabla 2 se indican las categorías, los indicadores de análisis y los instrumentos. Para la reducción de datos se utilizó una muestra empírica de material y análisis de contenido.

Tabla 2

Categorías de análisis e instrumentos

\begin{tabular}{|c|c|c|}
\hline Categorías & Indicadores & Instrumentos \\
\hline Lectura y fichaje & $\begin{array}{l}\text { Selección de autores } \\
\text { Número de documentos adecuados } \\
\text { Fichaje de acuerdo al modelo }\end{array}$ & $\begin{array}{l}\text { Trabajo de retroalimentación } \\
\text { Diario de campo } \\
\text { Entrega de matrices } \\
\text { Talleres de lectura } \\
\text { Participación en clases }\end{array}$ \\
\hline Selección de fuentes & $\begin{array}{l}\text { Fuente primaria } \\
\text { Fuente relacionada con el contenido a enseñar }\end{array}$ & $\begin{array}{l}\text { Trabajo de retroalimentación } \\
\text { Diario de campo }\end{array}$ \\
\hline $\begin{array}{l}\text { Transformación } \\
\text { del saber }\end{array}$ & $\begin{array}{l}\text { Vínculo con el curso } \\
\text { Vínculo con recursos de contexto }\end{array}$ & $\begin{array}{l}\text { Trabajo de retroalimentación } \\
\text { Presentación final } \\
\text { Rúbrica } \\
\text { Diario de campo }\end{array}$ \\
\hline $\begin{array}{l}\text { Manifestaciones del } \\
\text { pensamiento histórico }\end{array}$ & $\begin{array}{l}\text { Usa correctamente conceptos históricos. } \\
\text { Utiliza correctamente las nociones } \\
\text { de tiempo histórico } \\
\text { Establece relaciones causa-efecto }\end{array}$ & $\begin{array}{l}\text { Proceso de } \\
\text { Retroalimentación } \\
\text { Exposición } \\
\text { Respuesta a preguntas durante la exposición } \\
\text { Rúbrica }\end{array}$ \\
\hline $\begin{array}{l}\text { Comprensión } \\
\text { de la actividad }\end{array}$ & $\begin{array}{l}\text { Cumple los requerimientos del instructivo oral } \\
\text { y escrito }\end{array}$ & $\begin{array}{l}\text { Trabajo de retroalimentación } \\
\text { Exposición } \\
\text { Rúbrica }\end{array}$ \\
\hline
\end{tabular}

Fuente: elaboración propia

Los objetivos fueron:

a. Construir conocimientos en torno a la enseñanza de la historia a partir del uso de fuentes históricas en el aula.

b. Valorar el uso de las fuentes históricas como un recurso pedagógico para ser utilizado en el aula.

\section{Descripción de la actividad}

En la primera sesión, se identificó en forma oral y de manera grupal, el estado inicial del conocimiento de los estudiantes sobre fuentes históricas en el aula y la valoración que ellos hacen de estas frente a la enseñanza de la historia. Luego se profundizó en sesiones individuales el manejo de conceptos como fuentes históricas y pensamiento histórico.

En la segunda sesión, los estudiantes recibieron una clase teórica y textos para leer, que incluían definiciones, clasificación y tipos de fuentes históricas, y sus alcances teóricos en la enseñanza de la historia. Los estudiantes trabajaron en forma autónoma estos textos posteriormente. 
Durante la tercera sesión, los estudiantes debían presentar una fuente a trabajar, individualmente, en su diseño de clase. Tras esta elección, recibieron las siguientes instrucciones escritas (tabla 3) acompañadas de una rúbrica que evaluaría la exposición final (tabla 4). Ambos instrumentos orientaron el desarrollo de la actividad:

Tabla 3

Instrucciones de la actividad

\section{Instrucciones de la actividad}

a) Investigar sobre su utilidad y uso en la enseñanza de la Historia y Ciencias Sociales. (Se sugirió un número de 3 a 5 autores).

b) Determinar un contenido a partir del cual trabajar con la fuente histórica asignada. (El contenido fue uno de los trabajados en el curso donde desarrolló su proceso de práctica el presente semestre).

c) Proponer una secuencia de acciones didácticas para el tratamiento/enseñanza del contenido a partir de la fuente histórica asignada.

d) Diseñar una actividad pedagógica a partir de la fuente histórica asignada susceptible de ser aplicada en el curso en donde desarrolló su proceso de práctica.

e) Elaborar una planificación que dé cuenta de las diferentes acciones a desarrollar en torno al tratamiento de la fuente histórica y la enseñanza del contenido.

Fuente: elaboración propia

Tabla 4

Rúbrica de evaluación

\begin{tabular}{l|c|l}
\hline Criterio & Puntaje & Descripción \\
\hline Excelente & 4 & $\begin{array}{l}\text { La dimensión a evaluar cumple a cabalidad los aprendizajes esperados en el } \\
\text { desarrollo de la asignatura. }\end{array}$ \\
\hline Regular & 2 & $\begin{array}{l}\text { La dimensión a evaluar cumple parcialmente los aprendizajes esperados en el } \\
\text { desarrollo de la asignatura. }\end{array}$ \\
\hline Deficiente & 0 & La dimensión a evaluar no cumple los aprendizajes esperados en la asignatura. \\
\hline \hline
\end{tabular}

Indicadores

Puntaje

Aspectos formales (20\%)

\begin{tabular}{l|l}
\hline 1. Postura corporal, contacto visual y vestuario & \\
\hline 2. Vocabulario y comunicación & \\
\hline 3. Apoyo de recursos didácticos & \\
\hline
\end{tabular}

\section{Aspectos de fondo (80\%)}

1. Presenta evidencias (autores) sobre la utilidad y uso de la fuente histórica asignada en la enseñanza de la historia y ciencias sociales.

2. Propone una secuencia de acciones didácticas para el tratamiento/enseñanza del contenido a partir de la fuente histórica asignada.

3. Diseña una actividad pedagógica a partir de la fuente histórica asignada susceptible de ser aplicada en el curso en donde desarrolló su proceso de práctica.

4. Elabora una planificación que da cuenta de las diferentes acciones a desarrollar en torno al tratamiento de la fuente histórica y la enseñanza del contenido.

5. Dominio al exponer el tema

6. Aporte a la formación inicial

7. Capacidad de respuesta sobre el tema

Fuente: elaboración propia 
Todos los estudiantes debieron desarrollar lo anterior en dos sesiones durante las cuales fueron retroalimentados permanentemente por el profesor.

En el proceso se realizó una reflexión sobre la elección de las fuentes y su aplicación como estrategia de enseñanza en diversos contextos. Esto se enriqueció pues los avances se presentaron en forma abierta, lo que permitió que todo el grupo participara de las retroalimentaciones y explicaciones de parte del estudiante y del profesor.

En la quinta sesión, los estudiantes debieron exponer los resultados alcanzados en el desarrollo de las actividades realizadas. Fueron evaluados individualmente por medio de la rúbrica mencionada.

Para finalizar la actividad, en la sexta sesión, los estudiantes participaron de una instancia de retroalimentación en la cual, junto al profesor responsable de la actividad, analizaron sus principales fortalezas y debilidades durante la actividad desarrollada, en un afán de mejora del proceso de formación didáctica de los estudiantes.

\section{Resultados}

El proceso consistió fundamentalmente en reflexionar sobre la enseñanza de la historia en diferentes contextos educacionales, a partir del uso de fuentes históricas. Por medio de estrategias como clases teóricas, talleres, exposiciones, instancias de retroalimentación individual y grupal, se llevó a cabo la reflexión pedagógica y se regularon las actividades de enseñanza y aprendizaje que articularon la participación de los estudiantes durante los diferentes momentos. Al tratarse de un muestreo empírico, aun cuando se inspeccionó la totalidad de la muestra, los resultados reflejan solo aquello significativo (Flick, 2004), lo cual explica la estructura y los contenidos de las tablas.

La tabla 5 presenta el estado inicial y final de la categoría trabajo con fuentes históricas, a partir de los indicadores considerados.

Tabla 5

Categorías de análisis del trabajo con fuentes históricas

\begin{tabular}{l|l|l}
\hline $\begin{array}{l}\text { Indicadores } \\
\text { de análisis }\end{array}$ & \multicolumn{1}{|c}{ Al comenzar la actividad } & \multicolumn{1}{c}{ Al finalizar la actividad } \\
\hline $\begin{array}{l}\text { Selección de } \\
\text { fuentes }\end{array}$ & $\begin{array}{l}\text { Pocas veces hay una adecuada vinculación de la } \\
\text { fuente seleccionada con el contexto del centro de } \\
\text { práctica. } \\
\text { Se opta por fuentes históricas tradicionales. } \\
\text { Confusión sobre fuentes primarias. }\end{array}$ & $\begin{array}{l}\text { Se logra establecer una adecuada vinculación con los } \\
\text { contextos escolares involucrados en la actividad. } \\
\text { Hay una mayor variedad en la selección de las fuentes. } \\
\text { Logran diferenciar los tipos de fuentes históricas. }\end{array}$ \\
\hline $\begin{array}{l}\text { Tratamiento } \\
\text { de la fuente } \\
\text { histórica en } \\
\text { el diseño }\end{array}$ & $\begin{array}{l}\text { Fallan en el seguimiento de instrucciones. } \\
\text { En la planificación hay dificultades al momento } \\
\text { de establecer los aprendizajes esperados e } \\
\text { indicadores de evaluación. }\end{array}$ & $\begin{array}{l}\text { Se observa una mejoría importante en el seguimiento } \\
\text { de las instrucciones. } \\
\text { Las planificaciones logran evidenciar una coherencia } \\
\text { entre objetivos, aprendizajes esperados e indicadores } \\
\text { de evaluación. } \\
\text { La fuente seleccionada es el eje articulador de las } \\
\text { actividades propuestas para la clase. }\end{array}$ \\
\hline $\begin{array}{l}\text { Manifestaciones sencia un papel protagónico de la } \\
\text { del pensamiento seleccionada dentro de las actividades } \\
\text { histórico }\end{array}$ & $\begin{array}{l}\text { Pocas veces se evidencian expresiones, discursos } \\
\text { yacciones que den cuentan del dominio de } \\
\text { habilidades asociadas al conocimiento histórico. }\end{array}$ & $\begin{array}{l}\text { Reelaboran o reformulan el conocimiento a partir del } \\
\text { trabajo con fuentes históricas. }\end{array}$ \\
\hline $\begin{array}{l}\text { Comprensión de } \\
\text { la actividad }\end{array}$ & $\begin{array}{l}\text { Requieren un permanente apoyo de los docentes; } \\
\text { repetición de instrucciones y ejemplificaciones. }\end{array}$ & $\begin{array}{l}\text { Fallas en el seguimiento de instrucciones y } \\
\text { debilidades en el uso de estrategias para } \\
\text { el desarrollo de las etapas iniciales de la } \\
\text { intervención. }\end{array}$ \\
\hline \hline
\end{tabular}

Fuente: elaboración propia 
Al inicio de la actividad se evidenció, en algunos estudiantes, la falta de compromiso con su propio proceso de aprendizaje y el de sus compañeros. Esto se observó específicamente en los atrasos a la llegada a clases, las escasas intervenciones en ellas, el no acatamiento de instrucciones, el incumplimiento de las tareas en los plazos establecidos, la falta de rigurosidad en el trabajo grupal y un deficiente fichaje de textos escritos. Por ello, hubo que repetir las instrucciones escritas en forma oral y en forma individual.

Los estudiantes necesitaron el apoyo permanente de los docentes para desarrollar las primeras etapas de las actividades. Manifestaron dificultades para la selección de las fuentes históricas, confusión entre fuentes primarias y secundarias, predominio de fuentes escritas sobre otras, falta de pertinencia de los contenidos a enseñar y una escasa vinculación con el contexto de los centros de práctica. Otras dificultades estuvieron relacionadas con la planificación de la clase, evidenciándose errores en la selección de aprendizajes esperados e indicadores de evaluación, como también en la asignación de los tiempos a las actividades propuestas para los diferentes momentos de la clase.

En la tercera sesión, hubo cambios importantes en el desempeño, actitud y comportamiento de los estudiantes. Estaban más conscientes respecto de sus responsabilidades y deberes, lo que favoreció una conducta positiva frente a las diferentes actividades y una mayor motivación. Eran más autónomos y capaces de seguir las instrucciones, y requerían menos apoyo de los docentes. Participaban activamente de las clases, realizaban preguntas, eran sistemáticos y rigurosos con su trabajo dentro y fuera de la sala de clases, asistían a las instancias de retroalimentación, lograban seleccionar correctamente las fuentes primarias, y avanzaban en el diseño de su clase. Al acercarse la fecha de la exposición final del trabajo, aumentó el interés por solicitar entrevistas con los profesores para aclarar dudas y recibir retroalimentaciones.

De acuerdo con los indicadores de la rúbrica, fue posible apreciar que en su exposición final los estudiantes presentaron los resultados de la actividad apoyados en recursos audiovisuales de prolija elaboración. Estos fueron un complemento a la exposición, con una adecuada ortografía y el uso de organizadores gráficos. A pesar del nerviosismo inicial, los estudiantes fueron capaces de exponer su diseño y propuesta de trabajo. La mayoría tuvo una buena postura, vistió un atuendo de aspecto muy profesional, estableció un contacto visual con todos en el salón y utilizó un vocabulario apropiado para la audiencia.

Todas las exposiciones estuvieron respaldadas con referentes teóricos, pertinentes y recientes, sobre la utilidad y el uso de las fuentes históricas en la enseñanza de la historia y las ciencias sociales. Los estudiantes escogieron las fuentes cercanas a sus propias experiencias como textos escritos, fotografías y pinturas. Las fuentes materiales fueron escasamente utilizadas y las fuentes orales estuvieron ausentes.

La tabla 6 resume las fuentes representativas seleccionadas por los estudiantes y las actividades asociadas a ellas.

La mayoría de los estudiantes demostró comprensión conceptual y práctica del tema, ya que ellos fueron capaces de levantar una propuesta de planificación y diseñar una secuencia de actividades para el tratamiento de un contenido histórico a partir de la fuente histórica seleccionada. Las propuestas resultaban viables de ser implementadas en una sala de clases y favorecían el trabajo de aprendizajes conceptuales, procedimentales y actitudinales con estudiantes de diferentes contextos. 
Tabla 6

Opciones preferentes seleccionadas por los estudiantes

\begin{tabular}{|c|c|c|c|c|}
\hline Fuente utilizada & Contenidos & Actividades & Dificultades & Fortalezas \\
\hline $\begin{array}{l}\text { Pintura de la Revolución } \\
\text { Francesa }\end{array}$ & $\begin{array}{l}\text { Causas de la } \\
\text { revolución }\end{array}$ & $\begin{array}{l}\text { Guía con preguntas } \\
\text { en data }\end{array}$ & No supo explicar & $\begin{array}{l}\text { Pertinente elección de } \\
\text { la pintura }\end{array}$ \\
\hline $\begin{array}{l}\text { Documento relativo } \\
\text { a las condiciones de } \\
\text { trabajo infantil durante } \\
\text { la Revolución Industrial }\end{array}$ & $\begin{array}{l}\text { Problemática y } \\
\text { consecuencias sociales } \\
\text { de la Revolución } \\
\text { Industrial }\end{array}$ & $\begin{array}{l}\text { Lectura de documento } \\
\text { y guía con preguntas }\end{array}$ & $\begin{array}{l}\text { Preguntas breves. } \\
\text { Mal uso del recurso }\end{array}$ & $\begin{array}{l}\text { Buena elección del } \\
\text { documento }\end{array}$ \\
\hline $\begin{array}{l}\text { Imagen de pintura } \\
\text { rupestre }\end{array}$ & $\begin{array}{l}\text { Identificar forma } \\
\text { de vida durante el } \\
\text { Paleolítico }\end{array}$ & $\begin{array}{l}\text { Presentación en } \\
\text { Power Point. Imagen y } \\
\text { preguntas asociadas }\end{array}$ & Ninguna & $\begin{array}{l}\text { Relación entre la } \\
\text { fuente y el contenido }\end{array}$ \\
\hline $\begin{array}{l}\text { Carta de Diego } \\
\text { Portales }\end{array}$ & $\begin{array}{l}\text { Identificar al personaje } \\
\text { y su influencia en la } \\
\text { historia de Chile }\end{array}$ & $\begin{array}{l}\text { Guía de aprendizaje } \\
\text { asociada a la carta }\end{array}$ & Ninguna & $\begin{array}{l}\text { Relación entre la } \\
\text { fuente y el contenido } \\
\text { Buena construcción de } \\
\text { la guía }\end{array}$ \\
\hline $\begin{array}{l}\text { Extracto de la } \\
\text { Constitución de } 1933\end{array}$ & $\begin{array}{l}\text { Identificar la } \\
\text { importancia de esta } \\
\text { Constitución en el Chile } \\
\text { republicano }\end{array}$ & $\begin{array}{l}\text { Guía de aprendizaje } \\
\text { asociada a la carta }\end{array}$ & $\begin{array}{l}\text { Preguntas muy } \\
\text { sencillas. Poco } \\
\text { provecho de la fuente }\end{array}$ & $\begin{array}{l}\text { Buena elección de la } \\
\text { fuente en relación con } \\
\text { el contenido }\end{array}$ \\
\hline $\begin{array}{l}\text { Fotografía de la Guerra } \\
\text { de trincheras }\end{array}$ & $\begin{array}{l}\text { Etapas de la Segunda } \\
\text { Guerra Mundial }\end{array}$ & $\begin{array}{l}\text { Preguntas tras la } \\
\text { observación de la } \\
\text { imagen fotográfica }\end{array}$ & Ninguna & $\begin{array}{l}\text { Relación de la fuente } \\
\text { con el contenido. } \\
\text { Fotografía con } \\
\text { mucha información. } \\
\text { Preguntas bien } \\
\text { diseñadas. }\end{array}$ \\
\hline $\begin{array}{l}\text { Extractos de El martillo } \\
\text { de los brujos, de } \\
\text { Heinrich Kramer y } \\
\text { Jacobus Sprenger (1486) }\end{array}$ & $\begin{array}{l}\text { La crisis de la Baja Edad } \\
\text { Media en los siglos } \\
\text { XIV y XV: brujería y el } \\
\text { poder de la Iglesia }\end{array}$ & $\begin{array}{l}\text { Lectura y análisis del } \\
\text { texto mediante la } \\
\text { técnica iPreguntémosle } \\
\text { a la fuente! }\end{array}$ & Ninguna & $\begin{array}{l}\text { Habilidades } \\
\text { involucradas en las } \\
\text { actividades propuestas }\end{array}$ \\
\hline $\begin{array}{l}\text { Très riches heures du } \\
\text { duc de Berry (Las muy } \\
\text { ricas horas del duque de } \\
\text { Berry); calendarios }\end{array}$ & $\begin{array}{l}\text { Europa en la Edad } \\
\text { Media (siglos V-XIII); } \\
\text { sociedad feudal }\end{array}$ & $\begin{array}{l}\text { Elaboración de } \\
\text { un mural }\end{array}$ & Ninguna & $\begin{array}{l}\text { Transposición } \\
\text { didáctica }\end{array}$ \\
\hline $\begin{array}{l}\text { Pintura sobre el asalto a } \\
\text { la Bastilla }\end{array}$ & $\begin{array}{l}\text { Estado moderno (siglo } \\
\text { XVI-XVIII): Revolución } \\
\text { Francesa }\end{array}$ & Guía de actividades & $\begin{array}{l}\text { Las actividades no } \\
\text { favorecen el trabajo de } \\
\text { habilidades cognitivas } \\
\text { superiores }\end{array}$ & $\begin{array}{l}\text { Tratamiento del } \\
\text { contenido a partir de } \\
\text { la fuente seleccionada }\end{array}$ \\
\hline
\end{tabular}

Fuente: elaboración propia

Los estudiantes contestaron con precisión las preguntas formuladas por los profesores, compañeros de clase e invitados. Se comprobó que la totalidad de los estudiantes se basó en datos empíricos para diseñar su trabajo: de hecho, solo tres estudiantes habiendo diseñado la actividad no fueron capaces de justificar, frente a la comisión examinadora, las acciones propuestas y sustentar teóricamente sus argumentos.

Durante la retroalimentación final de la actividad, hubo un reconocimiento del valor — y la dificultad — del trabajo con fuentes en la enseñanza de la historia. Algunos de los comentarios de los estudiantes fueron: "las fuentes visibilizaban el pasado", "acercan el pasado al presente", "permiten ver restos del pasado", "fue una actividad exigente que nos permitió aprender acerca de cómo enseñar historia utilizando diferentes tipos de fuentes", "entendí para qué usar fuentes primarias", "las imágenes son superútiles para enseñar la Edad Media", "no se me ocurrió cómo usar esta imagen", "me costó encontrar más referentes teóricos que sustentaran mis actividades", "fue una actividad desafiante que nos obligó a usar fuentes históricas", "esta actividad nunca la hice en el liceo". 


\section{Discusión}

El uso de fuentes históricas primarias en el aula lleva a reflexionar sobre su función en la formación didáctica y disciplinar de los estudiantes de Pedagogía en Historia. En ese sentido, en la formación inicial se debe acompañar al futuro profesor en el desarrollo de habilidades asociadas al uso de fuentes, para incentivar de esta forma el desarrollo del pensamiento histórico acorde con su trabajo futuro en el aula. Lo anterior coincide con lo planteado por Pamela L. Grossman, Suzzane M. Wilson y Lee S. Shulman (2005) sobre el rol del profesor experto que conoce profundamente la materia y la sabe enseñar, en contraposición con el profesor que no domina los conocimientos disciplinares, lo que afecta el aprendizaje de sus estudiantes.

Al respecto, el rol del profesor experto resulta fundamental para el éxito de toda experiencia pedagógica, puesto que es quien planifica y acompaña en las acciones que llevarán al estudiante a ir construyendo su aprendizaje. Asimismo, la retroalimentación permanente y los instrumentos de evaluación que se diseñen resultan claves para generar un proceso de diálogo entre el profesor y los estudiantes en el cual se analicen las acciones desarrolladas por estos y se busque la mejora a partir del error. Tal como sostiene César Coll-Salvador (1990), la función del profesor no se limita solo a crear condiciones óptimas para que el estudiante despliegue una actividad mental constructiva, sino que debe orientar y guiar dicha actividad.

Sobre la actividad puntual con fuentes históricas en el aula, esta permitió a los estudiantes desarrollar competencias propias de la disciplina, para así vincular el pasado con el presente, desde sus propios intereses y motivaciones. Estudios como el de Augusta Valle-Taiman (2011, p. 23) también demuestran que el trabajo con fuentes constituye una gran oportunidad para el desarrollo del pensamiento crítico porque motiva a los estudiantes a utilizarlas para resolver un problema con sus propios argumentos y aproximarse por medio de estas a la naturaleza del conocimiento histórico.

Desde la etapa inicial, el estudiante realiza el proceso de elección de la fuente, asume el papel de investigador, y deja de lado la pasividad que caracteriza el aprendizaje de la disciplina. Concordando con lo que señala Elina Rostan (2002), el trabajo con fuentes en el ámbito escolar permite recrear y reconstruir el trabajo del historiador. Esto es, plantear problemas, elaborar hipótesis, argumentar y aprender procedimientos propios de la disciplina y su correspondiente conceptualización.

Lo anterior también se relaciona con la capacidad de juzgar los hechos históricos del pasado con marcos morales del pasado, parte central del pensamiento histórico. Los resultados de este estudio coinciden con lo expuesto por Antoni Santisteban-Fernández (2010), quien señala que una de las finalidades más importantes de la historia es dotar al alumnado de una serie de instrumentos de análisis, de interpretación o de comprensión para construir su propio estudio del pasado y juzgar los hechos históricos en su contexto de origen.

La actividad de trabajo con fuentes históricas en formación inicial implica un avance en el planteamiento de nuevos enfoques en la enseñanza de la historia, y permite reconocer la precaria formación docente en este ámbito. Coincide con los postulados de Joaquín Prats y Joan Santacana (2011b), quienes afirman que existe una tendencia dentro del profesorado de promover una enseñanza de historia enunciativa, poco activa y como un saber cerrado y concluido.

\section{Conclusiones}

El trabajo con fuentes históricas es fundamental en la formación inicial de futuros profesores de historia. Esta investigación permitió la obtención de evidencias sobre el nivel de conocimientos en el trabajo con fuentes de los estudiantes participantes.

Acercó a estos al método utilizado por el historiador al desarrollar habilidades asociadas al pensamiento histórico.

La utilización de fuentes mejoró la comprensión de los estudiantes sobre cómo se construye el pasado al reconocer aspectos valiosos como la subjetividad histórica y, por ende, reconocer la importancia del trabajo con estas en los centros de práctica a los cuales concurren.

Es necesario fortalecer en los estudiantes los marcos teóricos y conceptuales y buscar instancias para que puedan realizar una reflexión pedagógica de la actividad realizada. El trabajo con fuentes debe ser una estrategia que se utilice con los estudiantes en formación desde los primeros años de ingreso a la carrera.

La presente investigación tiene como principales limitaciones, primero, el tamaño y especificidad de la muestra, lo que incide en la generalización de los resultados y, segundo, que el estudio se realizó en el contexto de un curso en el que los sujetos pueden ajustar sus respuestas a lo que se espera de ellos. Por ello, en las futuras investigaciones será necesario examinar el trabajo de los estudiantes con fuentes históricas en contextos auténticos mediante observaciones en el aula.

Igualmente, en investigaciones futuras se sugiere indagar sobre otros métodos para la enseñanza de la historia que estimulen el desarrollo del pensamiento histórico en los estudiantes y los acerquen a la comprensión del pasado, para triangular información y de esta forma identificar el impacto de la formación del 
pensamiento histórico en la enseñanza y aprendizaje de la historia.

Finalmente, se recomienda hacer un seguimiento a los estudiantes que fueron parte del estudio, con el objetivo de establecer el impacto del trabajo con fuentes históricas en su desempeño académico en otros cursos asociados a la temática.

\section{Sobre los autores}

Elizabeth Gloria Montanares-Vargas es profesora de historia, geografía y ciencias sociales. Magíster en ciencias sociales aplicadas. Académica de la Universidad Católica de Temuco, Chile. Sus líneas de investigación están orientadas a la formación inicial docente, didáctica de la historia, enseñanza de la historia local.

Daniel Rodrigo Llancavil-Llancavil es profesor de historia, geografía y educación cívica. Magíster en educación, mención currículo y evaluación. Académico de la Facultad de Educación, Universidad Católica de Temuco, Chile. Sus líneas de investigación se orientan a la formación inicial docente, didáctica de la historia y la geografía.

\section{Referencias}

Andelique, C. M. (2011). La didáctica de la historia y la formación docente: ¿qué profesor de historia necesitan las escuelas? Clío \& Asociados, La historia enseñada, 15, 256-269. Disponible en: http://www.memoria.fahce.unlp.edu.ar/art revistas/pr.5025/pr.5025.pdf

Arráez, M.; Calles, J. \& Moreno, L. (2006). La hermenéutica: una actividad interpretativa. Sapiens, Revista Universitaria de Investigación, 7 (2), 171-181. Disponible en: http://www.redalyc. org/articulo.oa?id $=41070212$

Asociación Médica Mundial, AMM (1964). Declaración de Helsinki, Principios éticos para las investigaciones médicas en seres humanos. Disponible en: http:// www.wma.net/es/30publications/10policies/b3/

Bain, R. B. (2005). "¿Ellos pensaban que la tierra era plana?" Aplicación de los principios de cómo aprende la gente en la enseñanza de la Historia en la Educación Secundaria. Disponible en: http://www.eduteka.org/ ComoAprendenLosEstudiantes.php

Barton, K. C. (2010). Historia y epistemología de las ciencias. Investigación sobre las ideas de los estudiantes acerca de la historia. Enseñanza de las Ciencias Sociales: Revista de Investigación, 9, 97-114. Disponible en: http://dialnet.unirioja.es/ servlet/articulo?codigo $=3299879$

Botto, A. (2011). Dimensión ética de la investigación cualitativa. Revista Gaceta de Psiquiatría Universitaria, 7 (4), 354-357. Disponible en:
http://revistagpu.cl/2011/GPU_Dic_2011_PDF/ Editorial.pdf

Coll-Salvador, C. (1990). Aprendizaje escolar y construcción del conocimiento. Barcelona: Ediciones Paidós.

Éthier, M. A.; Demers, S. \& Lefrançois, D. (2010). Las investigaciones en didáctica sobre el desarrollo del pensamiento histórico en la enseñanza primaria. Una panorámica de la literatura publicada en francés e inglés desde el año 1990. Enseñanza de las Ciencias Sociales: Revista de Investigación, 9, 61-74. Disponible en: http://www.redalyc.org/ pdf/3241/324127609007.pdf

Flick, U. (2007). Introducción a la investigación cualitativa. Madrid: Ediciones Morata.

Fuentes, C. (2004). Concepciones de los alumnos sobre la historia. Enseñanza de las Ciencias Sociales: Revista de Investigación, 3, 75-83. Disponible en: http://www.raco.cat/index.php/EnsenanzaCS/ article/view/126194

Grossman, P.; Wilson, S. \& Shulman, L. S. (2005). Profesores de sustancia: el conocimiento de la materia para la enseñanza. Profesorado. Revista de Currículum y Formación del Profesorado, 9 (2), 1-25. Disponible en: http://recyt.fecyt.es/index. php/profesorado/article/view/42676/24576

Henríquez, R. (2011). Aprender a explicar el pasado: el rol de causalidad y las evidencias históricas en el aprendizaje de la historia de alumnos chilenos. En Ramón López-Facal, Luis Velasco-Martínez, Víctor Manuel Santidrián-Arias \& Xosé Ángel Armas-Castro (coords.). Pensar históricamente en tiempos de globalización: Actas de I Congreso Internacional sobre Enseñanza de la Historia, Santiago de Compostela, 30 de junio-2 de julio de 2010, 91-98. Santiago de Compostela: Universidad de Santiago de Compostela. Disponible en: https://www.academia.edu/1783730/ Pensar_hist\%C3\%B3ricamente_en_tiempos_de_ globalizaci\%C3\%B3n_actas_de_I_Congreso_ Internacional_sobre_ense\%C3\%B1anza_de_la_ historia Recurso electr\%C3\%B3nico

Lee, P. \& Ashby, R. (2000). Progression in Historical Understanding among Students Ages 7 to 14. En Peter N. Stearns, Peter Seixas \& Sam Wineburg (eds.). Knowing, Teaching and Learning History: National and International Perspectives, 199222. New York: University Press.

Meschiany, T. (2013). Reflexiones en torno al pasado reciente y la cultura escolar. Notas sobre investigación y enseñanza. Revista de Educación, 4 (6), 37-55. Disponible en: http:// fh.mdp.edu.ar/revistas/index.php/r_educ/ article/viewFile/751/790 
Pagès i Blanch, J. (2003). Enseñar a enseñar historia: la formación didáctica de los futuros profesores de historia. En José Antonio Gómez-Hernández, María Encarna Nicolás-Marín (coords.). Miradas a la Historia: reflexiones historiográficas en recuerdo de Miguel Rodríguez Llopis, 155-178. Barcelona: Universidad Autónoma de Barcelona, UAB. Disponible en: https://www.um.es/campusdigital/ Libros/textoCompleto/historia/12pages.pdf

Pagès i Blanch, J. (2009). El desarrollo del pensamiento histórico como requisito para la formación democrática de la ciudadanía. Reseñas de Enseñanza de la Historia, 7, 69-91. Disponible en: http://pagines.uab.cat/joan_pages/sites/ pagines.uab.cat.joan_pages/files/2009_Pages_ Rese\%C3\%B1as_7.pdf

Perafán, A. (2013). Reflexiones en torno a la didáctica de la historia. Guillermo de Ockham, Revista Científica, 11 (2), 149-160.

Pluckrose, H. (2002). Enseñanza y aprendizaje de la historia. Madrid: Ediciones Morata.

Prats, J. (2000). Dificultades para la enseñanza de la historia en la educación secundaria: reflexiones ante la situación española. Revista de Teoría y Didáctica de las Ciencias Sociales, 5, 71-98. Disponible en: http://www.saber.ula.ve/ bitstream/123456789/23950/1/joaquin_prats.pdf

Prats, J. \& Santacana-Mestre, J. (2011a). Enseñar a pensar históricamente: la clase como simulación de la investigación histórica. En Joaquín Prats-Cuevas (coord.). Didáctica de la Geografía y la Historia, 67-87. Barcelona: Ministerio de Educación, Cultura y Deporte, Editorial Graó, Instituto de Recursos e Investigación para la Formación, IRIF.

Prats, J. \& Santacana-Mestre, J. (2011b). ¿Por qué y para qué enseñar Historia? En Leopoldo F. RodríguezGutiérrez \& Noemí García-García (coords.). Enseñanza y aprendizaje de la Historia en la Educación Básica, 18-68. Cuauhtémoc, México: Secretaría de Educación Pública, SEP. Disponible en: https://www.academia.edu/4115769/ Ense\%C3\%B1anza_y_aprendizaje_de_la_ Historia_en_la_Educaci\%C3\%B3n_B\%C3\%A1 1 sica

Prieto, J.; Gómez, C. \& Miralles, P. (2013). El uso de fuentes primarias en el aula y el desarrollo del pensamiento histórico y social. Una experiencia en Bachillerato. Proyecto Clío, History and History
Teaching, 39 (14 páginas). Disponible en: http:// clio.rediris.es/n39/articulos/historiasocial/ PrietoGomezMiralles.pdf

Rostan, E. (2002). El trabajo con fuentes en la clase de Historia. Revista de la Educación del Pueblo, 87, 14-18.

Ruedas, M. (2012). Ontología conductista del hecho educativo en futuros docentes. Diálogos Educativos, 12 (24), 28-54. Disponible en: http://dialnet. unirioja.es/servlet/articulo?codigo $=4156171$

Sáiz, J. (2014). Fuentes históricas y libros de texto en secundaria: una oportunidad perdida para enseñar competencias de pensamiento histórico. Ensayos. Revista de la Facultad de Educación de Albacete, 29 (1), 83-99. Disponible en: https:// www.revista.uclm.es/index.php/ensayos/article/ view/503/458

Santisteban, A. (2010). La formación de competencias de pensamiento histórico. Clío \& Asociados, La historia enseñada, 14, 34-56. Disponible en: http://www.memoria.fahce.unlp.edu.ar/art_ revistas/pr.4019/pr.4019.pdf

Shulman, L. S. (1987). Knowledge and Teaching: Foundations of the New Reform. Harvard Educational Review, 57 (1), 1-22. Disponible en: http://gse.buffalo.edu/fas/yerrick/ubscience/ UB_Science_Education_Goes_to_School/21C Literature_files/shulman,\%201987.pdf. Traducción castellana (2005): Conocimiento y enseñanza: fundamento de la nueva reforma. Profesorado. Revista de Currículum y Formación de Profesorado, 9 (2), 1-30. Disponible en: http:// recyt.fecyt.es/index.php/profesorado/article/ view/42831/24722

Suárez, M. (2010). Enseñanza de la Historia: viejos problemas y necesidad de un cambio. Reflexión de un alumno del Máster de Profesorado de Secundaria. Proyecto Clío, History and History Teaching, 36. Disponible en: http://clio.rediris.es/ n36/articulos/suarez.pdf

Valle, A. (2011). El uso de fuentes escritas en la enseñanza de la Historia. Análisis de textos escolares para tercero y cuarto de secundaria. Revista Educación, 20 (38), 81-106. Disponible en: http://revistas.pucp.edu.pe/index.php/educacion/ article/view/2604/2552

Wineburg, S. S. (2001). Historical Thinking and Other Unnatural Acts: Charting the Future of Teaching the Past. Philadelphia: Temple University Press. 